http://rev.med.panacea.unica.edu.pe

Rev méd panacea. 2014; 4(1): 8-12.

Recibido: 15 de Abril del 2014 | Aceptado: 24 de Abril del 2014 | Publicado: 30 de Abril del 2014

CONFICTO DE INTERES: NO DECLARADOS

ARTÍCULO ORIGINAL

\title{
Inicio temprano de relaciones sexuales en adolescentes escolares de la ciudad de Ica, 2014
}

\author{
Sofía Rengifo-Venegas ${ }^{1, a}$, Viviana Uribe-Godoy ${ }^{1, a}$, Katherine Yporra-Quijandría ${ }^{\text {1,a }}$ \\ Facultad de Medicina, Universidad Nacional San Luis Gonzaga. Ica, Perú ${ }^{1}$ \\ Médico cirujano ${ }^{a}$
}

\begin{abstract}
O bjetivo: Determinar la prevalencia y las principales motivaciones para el inicio temprano de las relaciones sexuales en los adolescentes escolares de la ciudad de Ica. Materiales y métodos: Se realizó un estudio transversal en 682 estudiantes del tercer al quinto año de secundaria de dos colegios nacionales de la ciudad de Ica durante el año 2014. Resultados: 125 (18,33\%) de los adolescentes habían tenido relaciones sexuales, la edad promedio de este inicio fue a los $14,38 \pm 0,13$ años. $11(4,87 \%)$ mujeres y $114(25,00 \%)$ varones ya habían tenido relaciones sexuales, con mayor proporción en los ateos o agnósti$\cos (10 ; 41,67 \%) .20(16,00 \%)$ adolescentes que no vivían con sus madres, $56(44,80 \%)$ que no vivían con sus papás, y $61(48,80 \%)$ que no vivían con hermanos tuvieron este problema, al igual que en $70(36,08 \%)$ de los consumían licor, en $27(47,37 \%)$ de los que consumían estupefacientes $(p=0,000)$ y en $48(41,03 \%)$ de los que consumían cigarrillos. Conclusiones: Dos de cada diez adolescentes habían iniciado relaciones sexuales y está se relacionó con el género, su religión, la convivencia con los padres o hermanos, las características del entorno social, las creencias sobre las consecuencias de las relaciones coitales, los programas preferidos en televisión, utilizar el tiempo libre para estar con su enamorado(a), el consumo de sustancias tóxicas, sus comportamientos, el antecedente materno de embarazo adolescente y el maltrato por algún familiar.
\end{abstract}

Palabras clave: adolescente, relación sexual, sexualidad (fuente: DeCS BIREME).

Autor corresponsal: Sofía Rengifo Venegas . Dirección: Urb. Ciudadela Magisterial, calle Los Eucaliptos N-1 Ica, Perú. Teléfono: (51) 972914456 . Correo electrónico: sofia_14_3@hotmail.com .

\section{INTRODUCCIÓN}

La adolescencia es un período de transición también tuvieron su primera relación sexual entre la niñez y la edad adulta. La Organi- con una desconocida y/u otra persona; sezación Mundial de la Salud (OMS) (1) define ñalando que el principal motivo de inicio de la adolescencia como "la etapa que trans- su primera relación sexual fue el acuerdo curre entre los 10 y 19 años. En este periodo mutuo (37,1\%), seguido por la curiosidad se da el despertar sexual que desencadena- $(22,8 \%$ y la ocasión (11,4\%). Amorán y cols. rá más adelante su maduración sexual, pero (8), observó que había una relación inversapor diversos factores cada vez tiende a bus- mente proporcional entre el grado de incar su iniciación sexual a edades más tempranas (2-5).

Para Pérez y cols. (6) el $82 \%$ de las adolescentes se inician sexualmente entre los 12 y los 19 años. Gamarra e lannacone (7)reporta que la edad promedio en ambos sexos fue a los 14,58 años (13 a 17 años), la mayoría de ellos $(60 \%)$ tuvieron su primera relación sex ual con su enamorado(a). En cambio, solo en los varones $(15,8 \%)$ se observó que
Collins y cols. (11), demostró que los adolescentes que vieron más contenido sexual al inicio del estudio eran más propensos a iniciar relaciones sexuales al año siguiente. Santana y cols. (12), hallaron que el consumo de sustancias tóxicas se asociaba a un inicio más temprano de las relaciones sexuales en la adolescencia. Necchi y Schufer (13) demostró que los jóvenes en su mayoría iniciaron su vida sexual con una meretriz. Dietrich (14) estima que el 50\% de los adolescentes menores de 17 años son activos sexualmente.

Los riesgos de una falta de protección sexual no sólo significan la transmisión de enfermedades de transmisión sexual y el VIH/ SIDA, sino también de embarazo no deseado, que en muchos casos llevan al aborto clandestino y ha sido responsable de la muerte de muchas adolescentes. En el Perú, según el Instituto Nacional de Estadística e Informática (INEI 2007) (15) se registró 2,5 millones de mujeres adolescentes, de las cuales $7,3 \%$ son madres. La encuesta Demográfica y de Salud Familiar (ENDES) 2011 (16) reporta que el 12,5\% quedó embarazada entre los 15 y 19 años, observándose una tendencia al aumento de la cifra respecto a años anteriores.

Como vemos, diversos estudios nacionales e internacionales muestran que la edad de inicio sexual ha disminuido considerablemente. No disponemos de estudios actualizados que determinen con precisión la edad de inicio sexual en los adolescentes de Ica. Por ello, nuestro estudio tiene como objetivo identificar la prevalencia y las principales motivaciones al inicio temprano de las relaciones sexuales en los adolescentes 
escolares de la ciudad de Ica y, así, contribuir con la actualización de los datos disponibles sobre dicho tema, para establecer recomendaciones sobre la intervención más costo-efectiva para resolver este problema en nuestra región.

\section{MATERIALES Y MÉTODOS}

Se realizó un estudio transversal. El universo estuvo conformado por los alumnos matriculados en los Colegios Nacionales San Luis Gonzaga y Antonia Moreno de Cáceres de Ica en el año 2014. En la muestra se incluyeron a 682 alumnos del tercer al quinto año de secundaria, entre los 13 y 19 años.

Se realizó una encuesta, haciendo uso de un cuestionario anteriormente aplicado por un estudio similar.

Las variables de estudio fueron: inicio de relaciones sexuales (edad y motivación), año de estudios, edad del adolescente, edad del padre, edad de la madre y edad de la madre al primer hijo, religión de adolescente, situación conyugal de padres, convivencia, características del entorno social, fuente de información de sexualidad, creencias sobre las consecuencias de las relaciones coitales, programas preferidos en televisión, actividades en los tiempos libres, consumo de sustancias tóxicas, comportamientos, antecedentes familiares de embarazo adolescente, maltrato familiar, así como las características de la relación con la madre y con el padre.

Para la determinación de la significancia estadística se usó el Coeficiente de Alfa de Cronbach.

Se trabajó con el programa SPSS ${ }^{\circledR} 21.0$ en el que se realizó el análisis estadístico obteniéndose las medidas de tendencia central para las variables numéricas (media y error estándar) y las medidas de distribución (número y porcentaje) para las variables categóricas.

\section{RESULTADOS}

$125(18,33 \%)$ de los adolescentes había iniciado relaciones sexuales, siendo la edad promedio de este inicio 14 años. La persona con quien se produjo el inicio precoz en mayor porcentaje $(61,6 \%)$ fue con su enamorado(a) (Figura 1).

La principal motivación para el inicio precoz fue el deseo de probar $(46,4 \%)$ y, en igual porcentaje, porque se dio la ocasión (Gráfico 2). Observamos también que, en los adolescentes que manifestaron haber iniciado relaciones sexuales, el $65,60 \%$ de ellos hizo uso de algún método anticonceptivo en la primera relación sexual. (Figura 2).

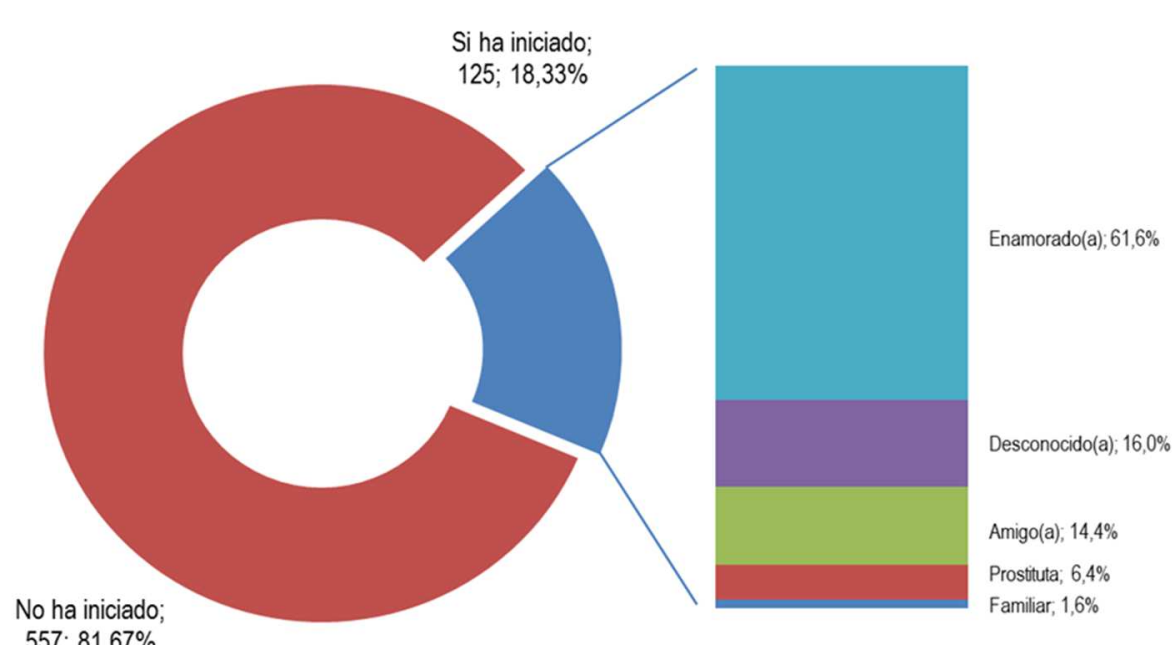

$557 ; 81,67 \%$

Figura 1. Prevalencia y persona con quien se produjo el inicio temprano de relaciones sexuales en adolescentes escolares de Ica, 2014.

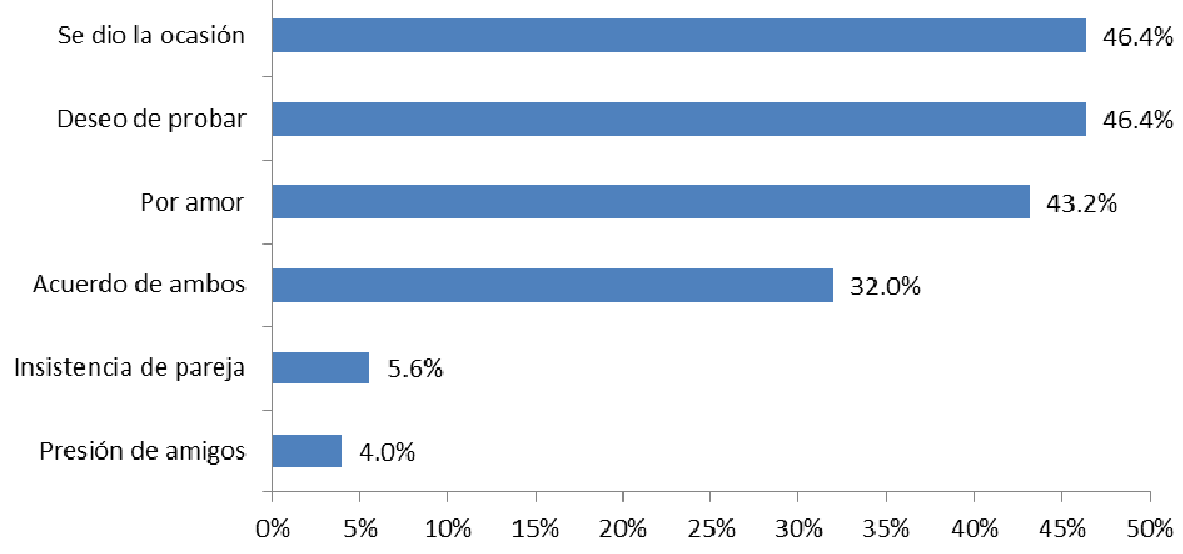

Figura 2. Motivación para el inicio temprano de relaciones sexuales en adolescentes escolares de Ica, 2014.

En cuanto al año de estudio, se observó que tenían una unión libre y en el 21,39\% no esta$13,99 \%$ de los que cursaban el tercer año, ban unidos

$20,00 \%$ de cuarto año y $23,67 \%$ de quinto año ya habían iniciado relaciones sexuales. Además el 4,87\% de las adolescentes de sexo femenino ya habían iniciado relaciones sexuales, mientras que en el sexo masculino este inicio temprano se observó en $25,00 \%$ de ellos.

El inicio precoz de relaciones sexuales se observó en mayor proporción en los adolescentes que manifestaron ser ateos o agnósticos (41,67\%). En aquellos que profesaban la religión católica se observaron $17,84 \%$, en los testigos de Jehová o mormones $15,63 \%$ y en los cristianos evangélicos $12,63 \%$. En aquellos que profesaban otras religiones se observaron $33,33 \%$.

En cuanto a la situación conyugal de los padres de los que ya habían iniciado relaciones sexuales, se observó que en el $14,47 \%$ los padres tenían una unión estable, en el 20,88\%
También en aquellos que iniciaron precozmente las relaciones sexuales, 35,20\% presencia de alcoholismo en la familia, $77,60 \%$ tenían enamorado(a) y 44,00\% tenían conocimiento que sus pares habían iniciado relaciones sexuales.

En relación al conocimiento de los adolescentes sobre las consecuencias de tener relaciones sexuales precozmente, $29,75 \%$ de los que mencionaron que las relaciones coitales nunca originan embarazo, $27,66 \%$ de los que pensaban que estas relaciones nunca trasmiten una ITS, $34,97 \%$ de los que señalaron que originaba dificultad para estudiar, $32,72 \%$ de los que creen que nunca lleva a decidir abortos y en $31,13 \%$ de los que no piensan que hace perder los estudios, ya existía práctica de relaciones sexuales. En relación a los programas de televisión preferidos estudiaban o trabajaban, $14,40 \%$ manifestaron 
por el adolescente, se observó que aquellos que veían películas de sexo tenían el mayor porcentaje $(46,67 \%)$ de quiénes habían iniciado relaciones sexuales precoces.

En relación a los hábitos nocivos, se observó inicio temprano de relaciones sexuales en $36,08 \%$ de los consumían licor, en $47,37 \%$ de los que consumían estupefacientes y en $41,03 \%$ de los que consumían cigarrillos. En aquellos que van a fiestas se observó que $20,80 \%$ ya había iniciado relaciones sexuales. Asimismo, en el grupo que manifiesta ver videos o revistas de sexo, 30,60\% de los adolescentes ya habían iniciado relaciones sexuales.

En el grupo de adolescentes con inicio temprano de relaciones sexuales, se observó que el antecedente familiar de embarazo adolescente fue materno en $37,60 \%$ y en el núcleo familiar en $11,20 \%$ En relación al maltrato familiar, en aquellos adolescentes que manifiestan haber sufrido violencia sexual por algún familiar, el $46,15 \%$ había iniciado relaciones sexuales, mientras que en aquellos que no sufrieron violencia sexual por algún familiar, se observó que $17,79 \%$ ya había iniciado relaciones sexuales.

\section{DISCUSIÓN}

En nuestro estudio, podemos determinar que aproximadamente dos de cada diez de los estudiantes adolescentes tiene un inicio temprano en sus relaciones sexuales, con un promedio de edad de 14 años, esta cifra es inferior a lo reportado por otros estudios (1719). Sin embargo, nuestros hallazgos son similares a los de Gamarra (20) cuya población es parecida a la nuestra, con la única diferencia de que esta provenía de colegios mixtos.

Encontramos que dos de cada tres adolescentes inicia su vida sexual con sus enamorados (as). Además, observamos que tener enamorado(s) y dedicar tiempo libre para estar con ellos (as), fue más frecuente en aquellos con inicio temprano de relaciones sexuales. Observamos que un tercio de ellos inicia estas relaciones con personas desconocidas o amigos, es decir, sin tener un vínculo sentimental u objetivo común de futuro. Este problema se magnifica cuando vemos que la motivación principal fue el deseo de conocer la experiencia, en casi la mitad de ellos. Siendo los varones y los que estaban en el último año de educación secundaria, los que presentaron mayor frecuencia de inicio de relaciones sexuales.

Encontramos cierta relacion entre la ausencia de la madre o padre o no tener o vivir con hermanos en el hogar y el inicio temprano de relaciones sexuales. Nuestros resultados son similares a los reportados por Chirinos (21) que señala que el nivel de educación de los padres no se asocia a la actividad sexual de los hijos.

Podemos observar que los programas que el adolescente prefiere ver en la televisión influyen en el inicio temprano de relaciones sexuales y cuando el programa que se prefiere, tiene contenido sexual, se presenta con mayor frecuencia este problema. De igual forma ocurre cuando el entrevistado menciona que ve videos o revistas con contenido sexual. Un hallazgo igual a lo reportado por Collins y cols. (11), quien demostró que los adolescentes que vieron más contenido sexual eran más propensos a iniciar relaciones sexuales al año siguiente.

El consumo de sustancias tóxicas (licor, estupefacientes o cigarrillos) fue más frecuente en aquellos con inicio temprano de relaciones sexuales. Aproximadamente tres a cinco de los adolescentes que consumen estas sustancias ya han iniciado relaciones sexuales. Igual influencia se observa cuando el adolescente asiste a fiestas, siendo similares estos resultados en otros estudios (22-23) También hallamos que la religión es un factor que se relaciona con el inicio precoz de relaciones sexuales siendo los ateos o agnósticos quienes presentan mayor porcentaje de inicio de relaciones sexuales.

Otro hallazgo importante de nuestro estudio, es que la presencia de maltrato de algún familiar (excepto padre o madre) y la violencia sexual contra el adolescente fue mayor en aquellos con inicio temprano de relaciones sexuales. Como ya se mencionó, la salud psicológica tiene una influencia muy importante en esta problemática, tal como lo señala Whitaker y cols. (4). Observamos también una que existe una relación significativa entre el antecedente familiar de embarazo adolescente en la madre, mas no en algún miembro del núcleo familiar.

En el currículo universitario actual (24) los temas de sexualidad son abordados como competencias transversales y directamente como contenidos de algunas asignaturas. Esta investigación nos permite coincidir con Rodríguez (25) quien menciona que en los colegios se dan lecciones de sexualidad con la misma rigurosidad metodológica y evaluativa de otras asignaturas. Rosas (26) señala que los adolescentes recurren preferentemente a los amigos para obtener información, que los padres y profesores les ocultan, o porque sus padres no poseen la información suficiente y por la desconfianza que sienten hacia ellos.

Asimismo, encontramos que las creencias sobre los riesgos a los que se expone el adolescente al tener relaciones coitales, influyen fuertemente en la iniciación sexual. Es decir, cuando el adolescente cree que no existen riesgos será mayor el número de ellos que iniciará tempranamente las relaciones sexuales. No observamos relación entre el inicio temprano de relaciones sexuales y utilizar el tiempo de ocio para conversar con los amigos, pero la presencia de prácticas de relaciones sexuales en los amigos cercanos al adolescente si influye en este inicio temprano. Esto se demostró en nuestro estudio, pues existía mayor frecuencia de inicio de relaciones sexuales en el grupo que conocían que sus amigos habían iniciado relaciones sexuales.

Finalmente, observamos que cuando el adolescente estudia y trabaja existe mayor frecuencia de inicio de relaciones sexuales. Esto puede deberse a que existe mayor autonomía y menor dependencia económica de los padres, lo que le permite acceder a sustancias tóxicas, participar de reuniones sociales (fiestas), entre otros.

\section{REFERENCIAS BIBLIOGRÁFICAS.}

1. Organización Mundial de la Salud. Organización Panamericana de la Salud. Recomendaciones para la acción. Asociación Mundial de Sexología, 2000. pp 5 - 23 [Citado en octubre 2009] [Link]

2. Guevara V. Impacto de un taller educativo en el nivel de conocimientos sobre sexualidad enfocado en la prevención del embarazo y las infecciones de transmisión sexual en adolescentes; 2002. [Citado: Enero 2009]. [Link]

3. Promotores Escolares para la Promoción de Estilos de Vida Saludables con énfasis en Sexualidad y Prevención de ITS/VIH y SIDA. Ministerio de Educación, Ministerio de Salud, CONAMUSA, Fondo Mundial, Universidad Cayetano Heredia, 2008.

4. Whitaker D, Miller K, y Clark L. Reconceptualizing adolescent sexual behaviour: beyong did they or didn't they?. Family Planning Perspectives, 2000; 32:111-117.

5. García P y col. Estudio diagnóstico en Adolescentes en el Perú: comportamientos sexuales y acceso a condón. Unidad de ETS/SIDA- Universidad Peruana Cayetano Heredia, 2005.

6. Pérez F, De la Quintana A, Hidalgo C, Dourojeani D. Sexualidad y mujeres jóvenes. Negociación, protección y placer. IES, Lima. 2003

7. Acosta D, Gastelo R. Embarazo en la Adolescencia y Proyecto de vida. Caso: 
Liceos Públicos de Cumaná, Municipio Sucre, Estado: Sucre, año Escolar 20102011. Venezuela, 2012.

8. Amoran O, Onadeko M y Adeniyi J. Parental influence on adolescent sexual initiation practices in Ibadan, Nigeria. Internacional Quaterly of Community Health Education, 2004-2005; (23): 7381.

9. Bachanas $\mathbf{P}$, Morris $M$, Lewis-Gess J, Sarett-Cuasay E, Sirl K, Ries J.K y Sawyer $\mathbf{M}$. Predictors of risky sexual behavior in African American adolescent girls: implications for prevention interventions. Journal of Pediatric Psychology. 2002; (27): 519-530.

10. Diez-Canseco F, Carrasco C. y Cueto S. Características de la salud sexual y reproductiva de las adolescentes de la comunidad afectada por violencia política. Huanta, Ayacucho. Informe de Investigación línea de base para el proyecto de Salud Sexual y Reproductiva en adolescentes afectados por la violencia política. Salud sin límites. 2000; pp: 57-61.

11. Collins R, Elliott M, Berry S, Kanouse D, Kunkel D, Hunter S, Miu A. Watching sex on television predicts adolescent initiation of sexual behavior. Pediatrics 2004; 114: 280-289.

12. Santana P, Verdeja V, Ovies C, Fleitas R. Asociación entre algunos factores psicosociales y el inicio de las relaciones sexuales en adolescentes escolares. Revista Cubana de Medicina General e Integrada, 2006; 22: 1-7.

13. Necchi S, Schufer M. Adolescente varón: iniciación sexual y anticoncepción (Argentina). Revista Chilena de Pediatría, 2001; 72: 159-168.

14. Dietrich JE. Adolescent sexuality: is your practice teen friendly?. Sexuality, Reproduction \& Menopause, 2009; 7: 17 $-21$.

15. Instituto de Estadística e Informática (INEI). Censo Nacional XI Población y Vivienda. Datos Demográficos del Perú, 2007. [Link]

16. Instituto Nacional de Estadística e Informática (INEI). Encuesta Demográfica y de Salud Familiar - ENDES 2011. Fecundidad. Lima 2012. pp 83 - 96

17. Loli A. La sexualidad en la adolescencia: Un estudio peruano. SMMISA- The Pathfinder Fund. Lima, 1986.

18. Sánchez V. Conocimientos y actitudes acerca de las ITS y el VIH/SIDA de los adolescentes varones en dos comunidades zonales de Chimbote-Perú. 2011; 2(1):161-167.

19. Chirinos J. Nivel de conocimientos, actitudes y prácticas asociadas al comportamiento sexual de riesgo en escolares adolescentes de dos colegios de la USE 06, Lima-Norte, Tesis para obtener el Doctorado de la Facultad de Psicología. Escuela de posgrado. Universidad Peruana Cayetano Heredia. Lima, 1996.

20. Gamarra-Tenorio P, Iannacone J. Factores asociados que influyen en el inicio de actividad sexual en adolescentes escolares de Villa San Francisco-Santa Anita, Lima-Perú. Revista The Biologist. Lima, 2010; 8(1): 55-70

21. Chirinos J, Brindis C, Salazar V, Bardales O. y Reátegui L. Perfil de las estudiantes adolescentes sexualmente activas en colegios secundarios de Lima, Perú. Rev Med Hered.1999;(10): 49-59.

22. Gamez A, García J, Martínez J. Factores asociados al inicio de relaciones sexuales en adolescentes de 14 a 17 año. Perú. Revista de la Facultad de Medicina UNAM. 2007;50 (2): 80-83.

23. González P, Garcés A, Rodas P, Divasto J. Factores psicosociales presentes en el consumo de bebidas alcohólicas en adolescentes de 15 a 19 años del $1^{\circ}$ al $5^{\circ}$ semestre del programa de enfermería en la CU.R.N. de la ciudad de Cartagena. Colombia, 2013.

24. Ministerio de Educación del Perú. Diseño Curricular Nacional de la Educación Básica Regular. Normas y orientaciones para el inicio del año escolar 2014. Lima, 2013.

25. Rodríguez T, Farrín M, Bonilla P. La cultura sexual de los adolescentes. Ediciones Abya - Yala. Quito, 1995.

26. Rosas Carmen. Tesis: "Las actitudes de los adolescentes hacia la sexualidad humana y las relaciones coitales". Arequipa, 1995. 
FINANCIAMIENTO: Autofinanciado

CONTRIBUCIONES DEAUTORÍA: SRV, VUG Y KYQ participaron en el diseño del estudio, el análisis de los datos, revisaron críticamente el artículo y aprobaron la versión final.

\section{Early initiation of sexual intercourse in a School in the city of Ica, 2014}

bjective: To determine the prevalence and the main reasons for early sex in school adolescents in
the city of Ica start. Methods: A cross sectional study was conducted in 2014 . Results 682 students
from third to fifth year of high school to two national schools in the city of Ica in the year $125(18.33 \%)$ of
the adolescents had had sex, the average age of the onset was $14.38 \pm 0.13$ for years. $11(4.87 \%)$ women and
$114(25.00 \%)$ males had had sex, with a greater proportion of atheists and agnostics $(10 ; 41.67 \%) .20(16.00 \%)$
adolescents not living with their mothers, $56(44.80 \%)$ who did not live with their parents, and $61(48.80 \%)$
who did not live with sister had this problem, as in $70(36.08 \%)$ of liquor consumed, in $27(47.37 \%)$ of those
who used drugs ( $(p=0.000)$ and $48(41.03 \%)$ of those who smoked cigarettes. Conclusions: Two out of ten
adolescents had initiated sex and is related to gender, religion, living with parents or siblings, the charac-
teristics of the social environment, beliefs about the consequences of sexual intercourse, preferred pro-
grams television, use the time off to be with her lover (a) the substance use, behavior, maternal history of
teen pregnancy and abuse by family members.

Keywords: teen, sexual relationship, sexuality . (source: MeSH NLM)

\section{CITA SUGERIDA.}

Rengifo-Venegas S, Uribe-Godoy V, Yporra-Quijandría K. Inicio temprano de relaciones sexuales en adolescentes escolares de la ciudad de Ica, 2014. Rev méd panacea.2014; 4 (1):8-12. 